\title{
On the Computation of Integrals of Bivariate Gaussian Distribution
}

\author{
Vincent Savaux and Luc Le Magoarou \\ $b<>$ com, Rennes, France \\ emails: \{vincent.savaux,luc.lemagoarou\}@b-com.com
}

\begin{abstract}
This paper deals with the computation of integrals of centred bivariate Gaussian densities over any domain defined as an angular sector of $\mathbb{R}^{2}$. Based on an accessible geometrical approach of the problem, we suggest to transform the double integral into a single one, leading to a tractable closed-form expression only involving trigonometric functions. This solution can also be seen as the angular cumulative distribution of bivariate centered Gaussian variables $(X, Y)$. We aim to provide a didactic approach of our results, and we validate them by comparing with those of the literature.

Index Terms-Bivariate Gaussian distribution, Digital communications, Signal processing, PSK
\end{abstract}

\section{INTRODUCTION}

The problems of estimating unknown parameters from noisy (disturbed) Gaussian observations and characterizing the performance of estimators are ubiquitous in a vast majority of quantitative fields. They have been thoroughly studied in an unified way by detection and estimation theory [1]-[3], and are frequently met in signal processing and digital communications [4], [5] as well. The problems involving Gaussian observations usually lead to the computation of multiple integrals of multivariate Gaussian distributions. However, these integrals generally do not admit closed-form solutions, and are costly to approximate.

In this paper, we focus on integrals of dimension two, i.e. we consider integrals of bivariate Gaussian densities over any angular sector of $\mathbb{R}^{2}$. This problem has been widely tackled in literature [6]-[8], but leads to simple specific solutions or intractable general solutions. Indeed, authors in [6], [7] propose a closed-form expression of the integral in the case where two correlated Gaussian variables have the same unitary variance, and the domain of integration is one quadrant of $\mathbb{R}^{2}$ (e.g. $\left(\mathbb{R}_{+}, \mathbb{R}_{+}\right)$). In [8], the distributions of the envelope and the phase of two correlated Gaussian variables are derived in a very general case of non-zero mean and non-unitary variance. However, these distributions have no closed-form nor tractable expressions, and the resulting probabilities even require the integration of these ones.

In the present paper, we tackle the problem of the integral of a bivariate Gaussian centred distribution over any angular sector of $\mathbb{R}^{2}$ delimited by two linear functions, and in the case where two correlated Gaussian variables have different variances. To do so, we use a geometrical approach to rewrite the double integral as a single one, leading to an accessible and tractable closed-form solution. To the best of our knowledge,

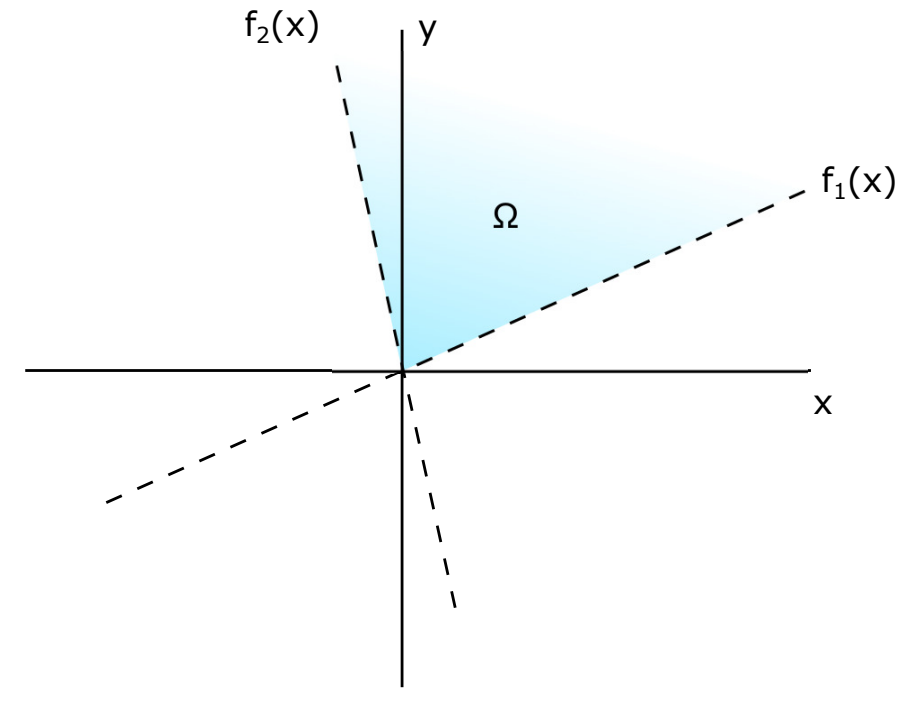

Fig. 1. Example of domain of integration $\Omega$.

this approach is original. Furthermore, this leads us to define an angular cumulative distribution function (CDF) of zeromean bivariate Gaussian variables. Our geometrical approach of the problem is didactically illustrated, and our results are compared with those of the literature [6]-[8].

The rest of the paper is organized as follows: Section II presents the general problem to be solved and gives the existing special solutions. In Section III, we derive the generalized solution, and Section V concludes this paper.

Notations: Vectors and matrices are respectively written in lower and upper case in boldface: $\mathbf{x}$, and $\mathbf{X}$. The scalars are written in normal font: $x$ or $X$. Moreover, $(.)^{T}$ and $|$.$| stand$ for the transpose and determinant operators, respectively, and $\mathbb{E}\{$.$\} for the mathematical expectation.$

\section{BACKGROUND AND COMMON RESULTS}

Let $X$ and $Y$ be two zero-mean Gaussian random variables with variances $\sigma_{x}^{2}=\mathbb{E}\left\{X^{2}\right\}$ and $\sigma_{y}^{2}=\mathbb{E}\left\{Y^{2}\right\}$, respectively. Moreover, the two variables $X$ and $Y$ are correlated, such that $\mathbb{E}\{X Y\}=\rho \sigma_{x} \sigma_{y}$, where $\rho \in[-1,1]$ is the so-called correlation coefficient. The joint probability density function of $(X, Y)$, denoted by $g_{X, Y}$, can be expressed as

$$
g_{X, Y}(\mathbf{x})=\frac{1}{2 \pi \sqrt{|\boldsymbol{\Sigma}|}} \exp \left(-\frac{1}{2} \mathbf{x}^{T} \boldsymbol{\Sigma}^{-1} \mathbf{x}\right),
$$


where $\mathbf{x}=(x, y) \in \mathbb{R}^{2}$, and $\boldsymbol{\Sigma}$ is the covariance matrix defined as

$$
\boldsymbol{\Sigma}=\mathbb{E}\left\{(X, Y)^{T}(X, Y)\right\}=\left(\begin{array}{cc}
\sigma_{x}^{2} & \rho \sigma_{x} \sigma_{y} \\
\rho \sigma_{x} \sigma_{y} & \sigma_{y}^{2}
\end{array}\right),
$$

such that $|\boldsymbol{\Sigma}|=\sigma_{x}^{2} \sigma_{y}^{2}\left(1-\rho^{2}\right)$. Computing any probability involving both $X$ and $Y$ (e.g. $\mathbb{P}(X \lessgtr 0 \cap Y \lessgtr 0)$ or $\mathbb{P}(X \lessgtr$ $0 \cup Y \lessgtr 0)$ ) requires the integral of (1) over subsets of $\mathbb{R}^{2}$. In the following, we consider the integral of $g_{X, Y}(\mathbf{x})$ over any angular sector in $\mathbb{R}^{2}$, and we define the integral

$$
\mathcal{M}=\iint_{\Omega} g_{X, Y}(\mathbf{x}) d x d y,
$$

where $\Omega$ is the angular sector which can be defined by

$$
\Omega=\left\{f_{1}(x) \lessgtr y \lessgtr f_{2}(x), x \in \mathbb{R}\right\},
$$

and $f_{1}(x)$ and $f_{2}(x)$ are linear functions such as illustrated in Fig. 1. Note that such angular divisions of $\mathbb{R}^{2}$ are usually considered in digital communications, for instance to define phase shift keying (PSK) modulations.

It must be reminded that a straightforward closed form of $\mathcal{M}$ is obtained when $\boldsymbol{\Sigma}$ is a scaled identity matrix. Moreover, a closed-form solution of the double integral (3) has been suggested in [6], [7] for any $\rho$ value, but only in the case where $\sigma_{x}^{2}=\sigma_{y}^{2}=1$ and $\Omega=\left(\mathbb{R}_{+}, \mathbb{R}_{+}\right)$. In that case, we obtain $\mathcal{M}=\frac{1}{4}+\frac{\arcsin (\rho)}{2 \pi}$. We hereby generalize the result to any case where $\sigma_{x}^{2}$ and $\sigma_{y}^{2}$ are non-unitary variances, as well as any domain of integration defined as $\Omega$. Moreover, this leads us to define an angular cumulative distribution function of bivariate Gaussian random variables.

\section{A Closed-Form Solution to (3)}

In this section, we derive a general solution of (3) in Theorem 1. To this end, let us first consider the change of basis $(x, y) \mapsto(\xi, \nu)$ where $(\xi, \nu)$ correspond to semi-axes of the ellipse defined by the equation

$$
(x, y)^{T} \boldsymbol{\Sigma}_{m, n}^{-1}(x, y)=1,
$$

such as illustrated in Fig. 2. This ellipse is a level set of the function $g_{X, Y}$. Without loss of generality, we assume that $\xi$ and $\nu$ correspond to the semi-major and semi-minor axes, respectively. Moreover, $\theta_{1}$ and $\theta_{2}$ are arbitrarily defined as the angles between the semi-major axis $\xi$ and the lines $f_{1}(x)$ and $f_{2}(x)$, respectively.

After the aforementioned change of basis, $\mathcal{M}$ in (3) can be rewritten as

$$
\mathcal{M}=\iint_{\Omega_{\xi, \nu}} \frac{1}{2 \pi \sigma_{\xi} \sigma_{\nu}} \exp \left(-\frac{1}{2}\left(\left(\frac{\xi}{\sigma_{\xi}}\right)^{2}+\left(\frac{\nu}{\sigma_{\nu}}\right)^{2}\right)\right) d \xi d \nu,
$$

where $\Omega_{\xi, \nu}$ is the new domain of integration of $\xi$ and $\nu$, which corresponds to the rotated angular sector $\Omega$ in the new basis. The standard deviations $\sigma_{\xi}$ and $\sigma_{\nu}$ are the length of

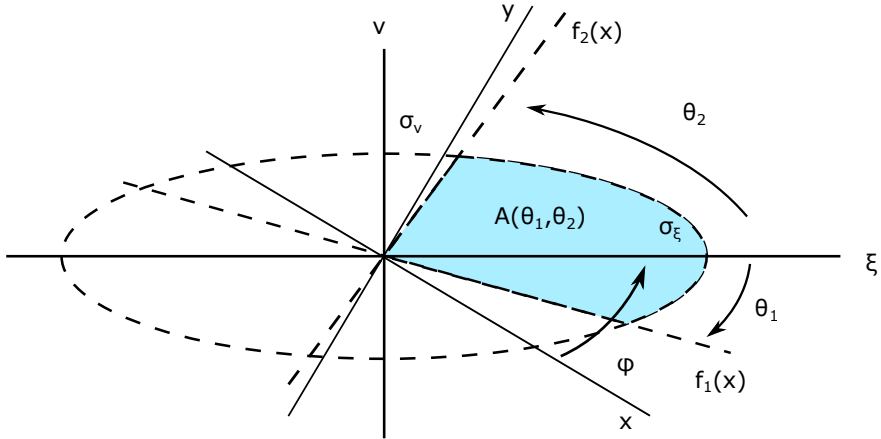

Fig. 2. Sector area of an ellipse in the general case.

the semi-major or semi-minor axes of the ellipse (5). Using the general ellipse equation and straightforward geometrical developments, they are expressed as

$$
\begin{aligned}
& \sigma_{\xi}=\left(\sigma_{x}^{2} \cos ^{2}(\varphi)+\sigma_{y}^{2} \sin ^{2}(\varphi)+\rho \sigma_{x} \sigma_{y} \sin (2 \varphi)\right)^{\frac{1}{2}} \\
& \sigma_{\nu}=\left(\sigma_{x}^{2} \sin ^{2}(\varphi)+\sigma_{y}^{2} \cos ^{2}(\varphi)-\rho \sigma_{x} \sigma_{y} \sin (2 \varphi)\right)^{\frac{1}{2}},
\end{aligned}
$$

where $\varphi$ is the angle between the axes $x$ and $\xi$, and is defined as

$$
\varphi= \begin{cases}\frac{1}{2} \arctan \left(\frac{2 \rho \sigma_{x} \sigma_{y}}{\sigma_{x}^{2}-\sigma_{y}^{2}}\right), & \text { if } \sigma_{x}^{2}>\sigma_{y}^{2} \\ \frac{\pi}{2}+\frac{1}{2} \arctan \left(\frac{2 \rho \sigma_{x} \sigma_{y}}{\sigma_{x}^{2}-\sigma_{y}^{2}}\right), & \text { if } \sigma_{x}^{2}>\sigma_{y}^{2} . \\ \frac{\pi}{4}, & \text { if } \sigma_{x}^{2}=\sigma_{y}^{2}\end{cases}
$$

Theorem 1. Let $p \in[0,1]$ be defined as the ratio of the semiminor and semi-major axes of the ellipse in (5), i.e. $p=\frac{\sigma_{\nu}}{\sigma_{\xi}}$. Then, for any $\theta_{1}, \theta_{2} \in[-\pi, \pi], \mathcal{M}$ can be expressed as

$$
\begin{aligned}
\mathcal{M} & =\iint_{\Omega} g_{X, Y}(\boldsymbol{x}) d x d y \\
& =\frac{Q\left(\theta_{1}, \theta_{2}\right)}{\pi},
\end{aligned}
$$

where $Q\left(\theta_{1}, \theta_{2}\right)$ is the function such that the area $A\left(\theta_{1}, \theta_{2}\right)$ of the elliptic sector bounded by $\left(\theta_{1}, \theta_{2}\right)$ is defined by $A\left(\theta_{1}, \theta_{2}\right)=\sigma_{\nu} \sigma_{\xi} Q\left(\theta_{1}, \theta_{2}\right)$. For simplicity purpose, we denote $\theta_{M}=\max \left(\left|\theta_{1}\right|,\left|\theta_{2}\right|\right)$ and $\theta_{m}=\min \left(\left|\theta_{1}\right|,\left|\theta_{2}\right|\right)$. Then, if $\theta_{1}, \theta_{2} \in\left[-\frac{\pi}{2}, \frac{\pi}{2}\right]$, we have

$$
Q\left(\theta_{1}, \theta_{2}\right)= \begin{cases}u\left(\left|\theta_{M}\right|\right)-u\left(\left|\theta_{m}\right|\right), & \text { if } \operatorname{sign}\left(\theta_{1}\right)=\operatorname{sign}\left(\theta_{2}\right) \\ u\left(\left|\theta_{M}\right|\right)+u\left(\left|\theta_{m}\right|\right), & \text { if } \operatorname{sign}\left(\theta_{1}\right) \neq \operatorname{sign}\left(\theta_{2}\right)\end{cases}
$$

where $u(\theta)=\frac{1}{2} \arctan \left(\frac{1}{p} \tan (\theta)\right)$. If $\theta_{1} \in\left[-\pi,-\frac{\pi}{2}\right] \cup\left[\frac{\pi}{2}, \pi\right]$ and $\theta_{2} \in\left[-\frac{\pi}{2}, \frac{\pi}{2}\right]\left(\theta_{1}\right.$ and $\theta_{2}$ are exchangeable $)$, then we get

$$
Q\left(\theta_{1}, \theta_{2}\right)=\left\{\begin{array}{l}
\frac{\pi}{2}-u\left(\pi-\left|\theta_{M}\right|\right)-u\left(\left|\theta_{m}\right|\right), \quad \text { if } \\
\operatorname{sign}\left(\theta_{1}\right)=\operatorname{sign}\left(\theta_{2}\right) \\
\frac{\pi}{2}-u\left(\pi-\left|\theta_{M}\right|\right)+u\left(\left|\theta_{m}\right|\right), \quad \text { if } \\
\operatorname{sign}\left(\theta_{1}\right) \neq \operatorname{sign}\left(\theta_{2}\right)
\end{array} .\right.
$$




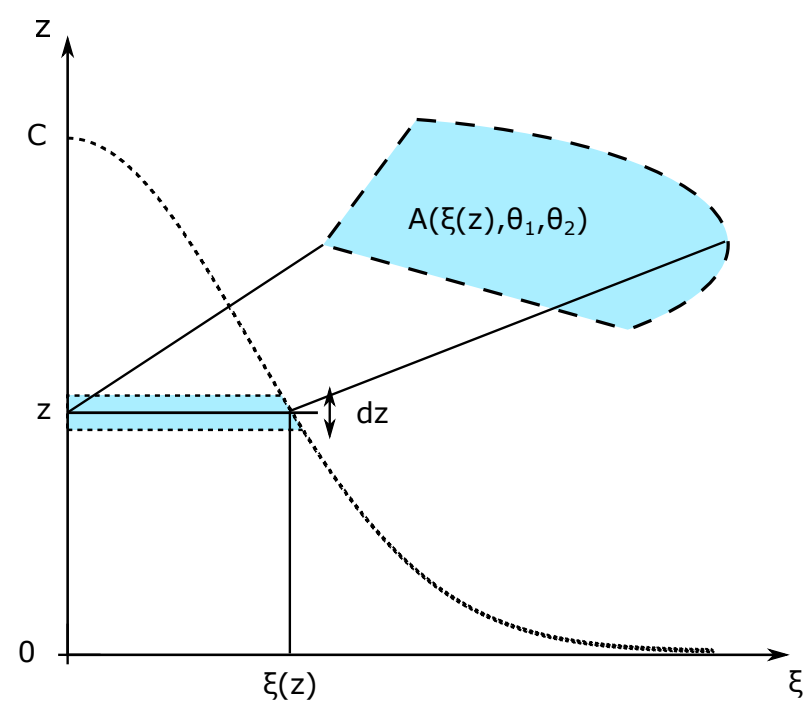

Fig. 3. The integral $\mathcal{M}$ can be computed by integrating $A\left(\xi(z), \theta_{1}, \theta_{2}\right)$ over the axis $z$.

Finally, if $\theta_{1}, \theta_{2} \in\left[-\pi,-\frac{\pi}{2}\right] \cup\left[\frac{\pi}{2}, \pi\right]$, we obtain

$$
Q\left(\theta_{1}, \theta_{2}\right)=\left\{\begin{array}{l}
u\left(\pi-\left|\theta_{m}\right|\right)-u\left(\pi-\left|\theta_{M}\right|\right), \quad \text { if } \\
\operatorname{sign}\left(\theta_{1}\right)=\operatorname{sign}\left(\theta_{2}\right) \\
\pi-u\left(\pi-\left|\theta_{M}\right|\right)-u\left(\pi-\left|\theta_{m}\right|\right), \quad \text { if } \\
\operatorname{sign}\left(\theta_{1}\right) \neq \operatorname{sign}\left(\theta_{2}\right)
\end{array} .\right.
$$

Proof. It must be emphasized that, although the expression of $\mathcal{M}$ seems to be more tractable in (6) than in (3), it can still not be computed in closed form, due to the complicated domains of integration. However, it can be noticed that the value $\mathcal{M}$ of the double integral corresponds to the volume under the curve

$$
g(\xi, \nu)=\frac{1}{2 \pi \sigma_{\xi} \sigma_{\nu}} \exp \left(-\frac{1}{2}\left(\left(\frac{\xi}{\sigma_{\xi}}\right)^{2}+\left(\frac{\nu}{\sigma_{\nu}}\right)^{2}\right)\right),
$$

in the angular sector $\Omega$ (or equivalently $\Omega_{\xi, \nu}$ ). This volume can also be assessed by summing (integrating) the areas $A$ of the elliptical sectors corresponding to the angular sector $\Omega$ (see Figs. 2 and 3). For any angles $\theta_{1}, \theta_{2}$ and any length of the semi-major axis $\xi$, given that $p=\frac{\nu}{\xi}$, the sector area $A\left(\xi, \theta_{1}, \theta_{2}\right)$ of the ellipse is given by

$$
A\left(\xi, \theta_{1}, \theta_{2}\right)=\xi \nu Q\left(\theta_{1}, \theta_{2}\right)=\xi^{2} p Q\left(\theta_{1}, \theta_{2}\right) .
$$

The expression of $A$ is proved in Appendix A. It follows from (13) that the sector area of the ellipse only depends of the angular sector defined by $\left(\theta_{1}, \theta_{2}\right)$, and the length $\xi$. By setting $z=g(\xi, \nu=0)$ and $\xi \in \mathbb{R}_{+}$, then for any $z \in\left[0, C=\frac{1}{2 \pi \sigma_{\nu} \sigma_{\xi}}\right]$ the following bijection holds:

$$
\begin{aligned}
\xi(z) & =g^{-1}(z) \\
& =\left(2 \sigma_{\xi}^{2} \ln \left(\frac{C}{z}\right)\right)^{\frac{1}{2}},
\end{aligned}
$$

allowing us to write $\xi \in[0,+\infty[$ in function of $z$. Such as aforementioned, $\mathcal{M}$ in (6) can then be rewritten as the integral of the sector areas $A\left(\xi(z), \theta_{1}, \theta_{2}\right)$ of the ellipse over $z \in\left[0, C=\frac{1}{2 \pi \sigma_{\nu} \sigma_{\xi}}\right]$ (see Fig. 3), which yields

$$
\mathcal{M}=\int_{0}^{C} A\left(\xi(z), \theta_{1}, \theta_{2}\right) d z
$$

Hence, considering the substitution $X=\ln \left(\frac{C}{z}\right)$ and $p=\frac{\sigma_{\nu}}{\sigma_{\xi}}$, we obtain

$$
\begin{aligned}
\mathcal{M} & =\int_{0}^{C} 2 p Q\left(\theta_{1}, \theta_{2}\right) \sigma_{\xi}^{2} \ln \left(\frac{C}{z}\right) d z \\
& =\int_{0}^{+\infty} 2 p Q\left(\theta_{1}, \theta_{2}\right) \sigma_{\xi}^{2} C e^{-X} d X \\
& =\frac{p Q\left(\theta_{1}, \theta_{2}\right) \sigma_{\xi}^{2}}{\pi \sigma_{\nu} \sigma_{\xi}} \\
& =\frac{Q\left(\theta_{1}, \theta_{2}\right)}{\pi},
\end{aligned}
$$

which concludes the proof.

It is worth mentioning that the suggested closed-form solution only involves the computation of the well-known trigonometric functions tan and arctan, and avoids the computation of the integral of the distributions in [8], or the double integral as in (3). In fact, the latter should usually be approximated by truncating discrete sums or by using a MonteCarlo method, both methods being computationally expensive. In the following, we provide general results related to the analytic solution of the integral $\mathcal{M}$, and we show that it reduces to known results given in [6], [7] in specific cases. Furthermore, an example of application is given as well.

\section{Results Related to $\mathcal{M}$}

\section{A. Angular Cumulative Distribution Function $\mathcal{M}$}

A first important result is that $\mathcal{M}$ in (9) can be interpreted as the angular cumulative distribution (CDF) of bivariate Gaussian variables $(X, Y)$. Thus, Fig. 4 illustrates $\mathcal{M}$ in function of $\left(\theta_{1}, \theta_{2}\right) \in[-\pi, \pi]^{2}$, for $p=\frac{1}{3}$. It can be observed that the $\operatorname{CDF} \mathcal{M}$ is symmetric with respect to the line $\theta_{2}=\theta_{1}$, for which $\mathcal{M}=0$, and that $\mathcal{M}=1$ for $\theta_{2}=-\theta_{1}= \pm \pi$. Note that this representation of the angular $\mathrm{CDF} \mathcal{M}$ depends on the values of $\rho, \sigma_{x}$, and $\sigma_{y}$ only through the $p$ value, since Fig. 4 holds in the basis $(\xi, \nu)$. Different behaviors of $\mathcal{M}$ would be obtained in the basis $(x, y)$, such as discussed afterward.

Alternatively to the two-dimensional representation of $\mathcal{M}$, Fig. 5 shows the $\operatorname{CDF} \mathcal{M}$ versus $\theta_{1} \in[-\pi, \pi]$ for a fixed $\theta_{2}=-\pi$. Note that in that case, the expression of $\mathcal{M}$ in (9)-(12) simplifies to:

$$
\mathcal{M}\left(\theta_{1}\right)=\left\{\begin{array}{ll}
\frac{u\left(\pi+\theta_{1}\right)}{\pi}, & \text { if } \theta_{1} \in\left[-\pi,-\frac{\pi}{2}\right] \\
\frac{1}{2}+\frac{u\left(\theta_{1}\right)}{\pi}, & \text { if } \theta_{1} \in\left[-\frac{\pi}{2}, \frac{\pi}{2}\right] \\
1-\frac{u\left(\pi-\theta_{1}\right)}{\pi}, & \text { if } \theta_{1} \in\left[\frac{\pi}{2}, \pi\right]
\end{array} .\right.
$$




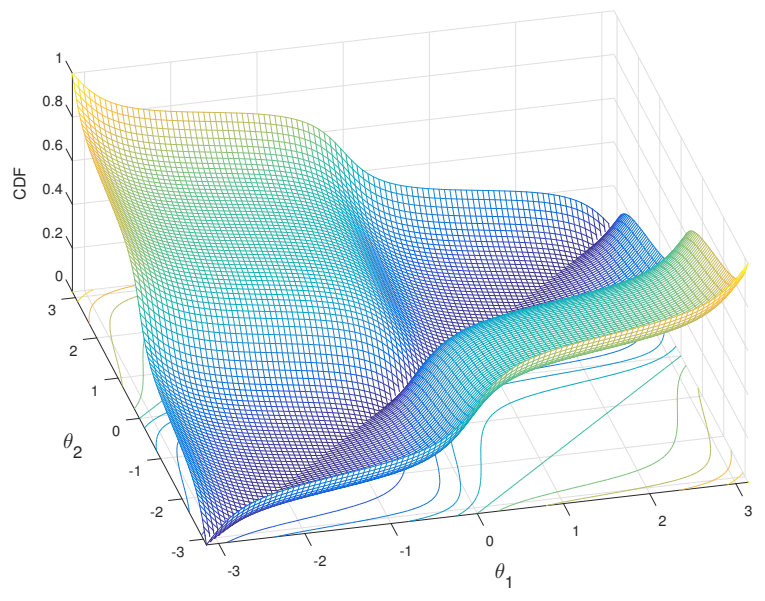

Fig. 4. $\mathcal{M}$ interpreted as the angular $\mathrm{CDF}$ of bivariate Gaussian variables $(X, Y)$, for $p=1 / 3$.

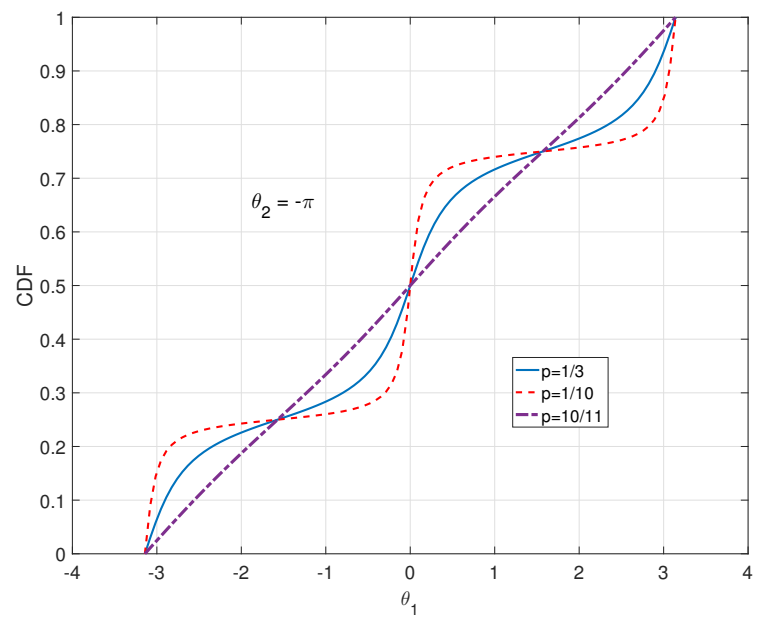

Fig. 5. Angular CDF $\mathcal{M}$ versus $\theta_{1} \in[-\pi, \pi], \theta_{2}=-\pi$. Comparison for different $p$ values.

Three behaviors of $\mathcal{M}$, taking $p \in\left\{\frac{1}{3}, \frac{1}{10}, \frac{10}{11}\right\}$ are shown in Fig. 5. It can be observed that $\mathcal{M}$ tends to the linear function $\mathcal{M}\left(\theta_{1}\right)=\frac{1}{2}+\frac{\theta_{1}}{2 \pi}$ when $p$ approaches 1 . This highlights the fact that $p=1$ corresponds to an uncorrelated couple of Gaussian variables $(X, Y)$ with $\sigma_{x}^{2}=\sigma_{y}^{2}$ (i.e. the ellipse (5) reduces to a circle), therefore having an uniformly distributed phase. Otherwise when $p$ approaches $0, \mathcal{M}$ approaches a step function, highlighting that the ellipse becomes a line.

\section{B. Particular Cases}

In this section we show that the general expression of $\mathcal{M}$ in (9)-(12) simplifies to known results given in [6], [7] when $\sigma_{x}^{2}=\sigma_{y}^{2}=1$ and when $\Omega$ is one of the four quadrants of $\mathbb{R}^{2}$. If we assume that $\rho \geq 0$ without loss of generality, we have $\varphi=\frac{\pi}{4}$ and then $p=\sqrt{\frac{1-\rho}{1+\rho}}$. Consider the quadrant $\left(\mathbb{R}_{+}, \mathbb{R}_{+}\right)$, then $\theta_{1}=-\frac{\pi}{4}$, and $\theta_{2}=\frac{\pi}{4}$, hence $\mathcal{M}$ in (9)-(10) becomes

$$
\mathcal{M}=\frac{1}{\pi} \arctan \left(\sqrt{\frac{1+\rho}{1-\rho}}\right) .
$$

Then, by using some relationship between trigonometric functions (e.g. the half-angle formula), we obtain the equivalent expressions:

$$
\begin{aligned}
\mathcal{M} & =\frac{1}{\pi} \arctan \left(\frac{1+\rho}{\sqrt{1-\rho^{2}}}\right) \\
& =\frac{1}{2}-\frac{1}{\pi} \arctan \left(\frac{\sqrt{1-\rho^{2}}}{1+\rho}\right) \\
& =\frac{1}{2}-\frac{1}{2 \pi} \arccos (\rho) \\
& =\frac{1}{4}+\frac{1}{2 \pi} \arcsin (\rho) .
\end{aligned}
$$

Similarly, it can be straightforwardly shown that if $\rho \geq 0$ and $\Omega=\left(\mathbb{R}_{+}, \mathbb{R}_{-}\right)$(or equivalently $\rho \leq 0$ and $\Omega=\left(\mathbb{R}_{+}, \mathbb{R}_{+}\right)$) we have $\mathcal{M}=\frac{1}{2 \pi} \arccos (\rho)$. These results also match those obtained by the integration of the PDF $f_{\theta}(\theta)=\frac{\sqrt{1-\rho^{2}}}{2 \pi(1-\rho \sin (2 \theta))}$ given in [8] (see (4) in [8] considering zero-mean variables):

$$
\int_{0}^{\frac{\pi}{2}} f_{\theta}(\theta) d \theta=\frac{1}{4}+\frac{1}{2 \pi} \arcsin (\rho) .
$$

Moreover, it must be noted that these expressions only depend on $\rho$, and we can show that this behavior holds for any variance of the variables $X$ and $Y$ as well. In fact, we directly have

$$
\mathcal{P}=\mathbb{P}(X \lessgtr 0 \cap Y \lessgtr 0)=\mathbb{P}\left(\frac{1}{\sigma_{x}} X \lessgtr 0 \cap \frac{1}{\sigma_{y}} Y \lessgtr 0\right),
$$

where $\frac{1}{\sigma_{x}} X$ and $\frac{1}{\sigma_{y}} Y$ have unitary variance. In the following, we show that the suggested general expression is mandatory to calculate more elaborate probabilities than (21).

\section{Application to More Elaborate Probabilities}

Let us consider zero-mean Gaussian variables $X$ and $Y$ with variances $\sigma_{x}^{2}$ and $\sigma_{y}^{2}$, respectively, and correlation coefficient $\rho$. For any $(\alpha, \beta) \in \mathbb{R}_{+}^{2}$, the suggested analysis allows us to calculate probabilities such as

$$
\mathcal{P}=\mathbb{P}(X \geq 0 \cap Y \geq 0 \cap \alpha Y \geq \beta X),
$$

where $\mathcal{P}$ is not independent of the variances of $X$ and $Y$ anymore due to the additional event $\alpha Y \geq \beta X$. To reduce the number of degrees of freedom of the problem, let us set $r=\frac{\sigma_{x}}{\sigma_{y}}$, and $\gamma=\frac{\beta}{\alpha}$. Then, the orientation $\phi$ and the ratio $p$ of the ellipse depends on both $\rho$ and $r$, and the domain of integration $\Omega$ (partially) depends on $\gamma$, such as illustrated in Fig. 6. Thus, in the example given in (22), we have $\theta_{2}=\frac{\pi}{2}$ and $\theta_{1}=\arctan (\gamma)$.

To better illustrate the dependence of $\mathcal{P}$ in (22) on the variances of $X$ and $Y$, we consider the following numerical application: $\rho=0.5, \gamma=1$, and $r \in\left\{\frac{1}{4}, 1,4\right\}$, and we imposed $\sigma_{x} \sigma_{y}=4$. Fig. 7 shows the level sets of the three 


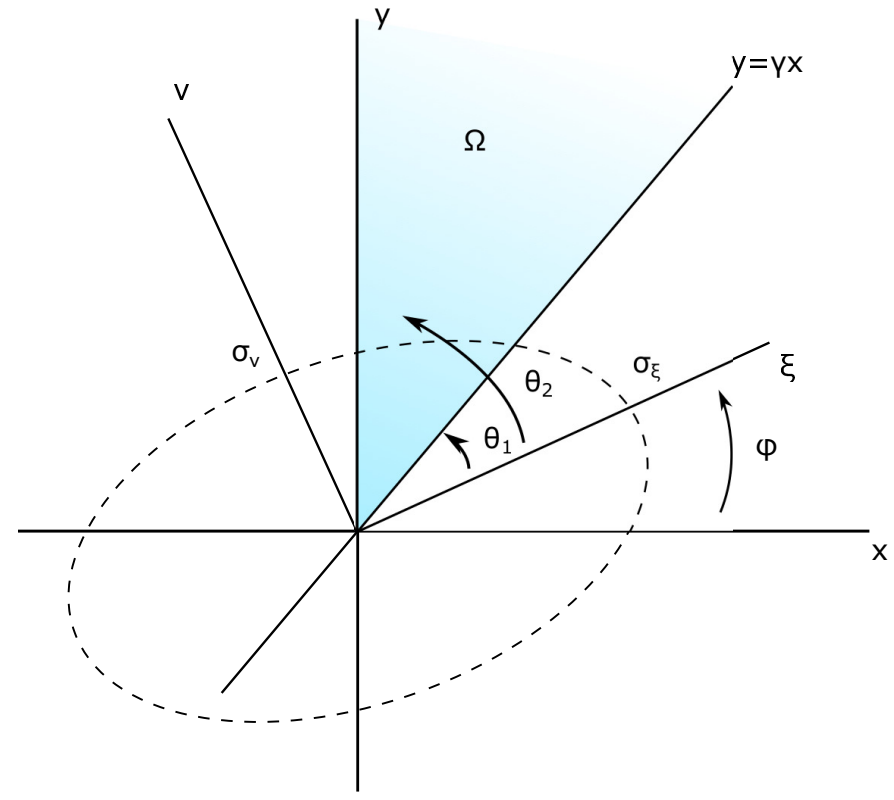

Fig. 6. Example of application for problems such as (22).

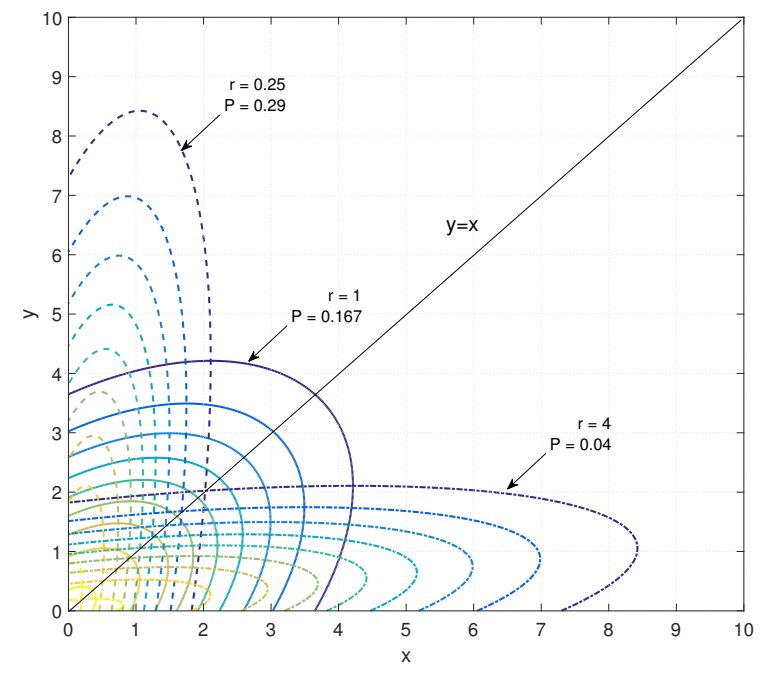

Fig. 7. $\mathcal{M}$ largely depends on the variances of $X$ and $Y$.

considered bivariate Gaussian distributions. The domain of integration $\Omega$ is above the line $y=x$. The corresponding values of probability $\mathcal{P}$ have been reported on Fig. 7, and it can be shown that the lower $r$, the larger $\varphi$ and therefore the larger $\mathcal{P}$, since the sector areas $A\left(\xi, \theta_{1}, \theta_{2}\right)$ of the ellipses in $\Omega$ are greater.

Such as mentioned in Section II and stated in [8], such results can be applied in the domains of digital communications and signal processing. For instance, it allows for an accurate characterization of the error rate of PSK-modulated signals with inphase/quadrature (I//Q) correlated noise. The main difference of the suggested analysis with results given in [8] is that we carry out our developments in the basis $(\xi, \nu)$ instead of $(x, y)$, leading to tractable closed-form expressions of $\mathcal{M}$, where the parameters $\sigma_{x}, \sigma_{y}$, and $\phi$ inherent to the basis $(x, y)$ are included in the geometrical parameter $p$ in the basis $(\xi, \nu)$.

To complete this study, non-zero means of $X$ and $Y$ could be included in a similar geometrical approach of the problem. Furthermore, this analysis could be extended in dimensions larger than two (involving ellipsoides), in order to tackle the problem of the integrals of multivariate Gaussian distributions.

\section{CONCLUSION}

In this paper we suggested a geometrical approach to solve the problem of integrating centered bivariate Gaussian densities over any domain defined as an angular sector of $\mathbb{R}^{2}$. This led to a tractable solution composed of trigonometric functions, which can be seen a the angular CDF of a couple of centered Gaussian variables $(X, Y)$. The developments were illustrated to provide a didactic approach of our results. Furthermore, we compared them to those of the literature in order to validate our solution. Future works will investigate the possible extension of the suggested geometrical approach to non-centred correlated Gaussian variables, as well as problems of dimension larger than two.

\section{APPENDIX}

\section{A. Proof of the expression of $A(\xi, \theta)$}

We consider an ellipse with semi-major and semi-minor axes $\xi$ and $\nu$, respectively. The area $A$ of the elliptical sector between the semi-major axis and the radius $r$ with angle $d \theta$ is equal to $A=\frac{r^{2}}{2} d \theta$. The parametric equation of the ellipse is expressed as

$$
\begin{aligned}
& x=\xi \cos (t) \\
& y=\nu \sin (t) .
\end{aligned}
$$

Thus, any radius with length $r$ and angle $\phi$ (corresponding to parameter $t=t_{\phi}$ ) with respect to the semi-major axis admits the equations:

$$
\begin{aligned}
\frac{y}{x} & =\tan (\phi)=\frac{\nu}{\xi} \tan \left(t_{\phi}\right) \\
\Rightarrow t_{\phi} & =\arctan \left(\frac{\xi}{\nu} \tan (\phi)\right),
\end{aligned}
$$

and then the substitution of $x$ and $y$ in $r^{2}=x^{2}+y^{2}$ yields

$$
r^{2}(\phi)=\left(\frac{\xi}{\sqrt{\frac{\xi^{2} \tan ^{2}(\phi)}{\nu^{2}}}}\right)^{2}+\left(\frac{\xi \tan (\phi)}{\sqrt{\frac{\xi^{2} \tan ^{2}(\phi)}{\nu^{2}}}}\right)^{2},
$$

which simplifies into

$$
r^{2}(\phi)=\frac{1}{\frac{\cos ^{2}(\phi)}{\xi^{2}}+\frac{\sin ^{2}(\phi)}{\nu^{2}}} .
$$

Hence we finally obtain 


$$
\begin{aligned}
& A(\theta)=\frac{1}{2} \int_{0}^{\theta} r^{2}(\phi) d \phi \\
& =\frac{\xi \nu}{2} \arctan \left(\frac{\xi}{\nu} \tan (\theta)\right),
\end{aligned}
$$

which concludes the proof.

\section{REFERENCES}

[1] E. L. Lehmann and J. P. Romano, Testing statistical hypotheses. Springer Science \& Business Media, 2006.

[2] E. L. Lehmann and G. Casella, Theory of point estimation. Springer Science \& Business Media, 2006.

[3] H. L. Van Trees, Detection, estimation, and modulation theory, part I: detection, estimation, and linear modulation theory. John Wiley \& Sons, 2004.

[4] S. M. Kay, "Fundamentals of Statistical Signal Processing: Estimation Theory". Prentice-Hall, 1998.

[5] — - "Fundamentals of Statistical Signal Processing: Detection Theory”. Prentice-Hall, 1998.

[6] C. Rose and M. D. Smith, Mathematical Statistics with Mathematica. Springer-Verlag, 2002, ch. 6: Multivariate Distributions, pp. 187 - 249.

[7] A. Stuart and J. K. Ord, Kendall's Advanced Theory of Statistics, Volume 1, Distribution Theory. Wiley, 2010, ch. 7: Multivariate Distributions.

[8] P. Dharmawansa, N. Rajatheva, and C. Tellambura, "Envelope and Phase Distribution of Two Correlated Gaussian Variables," IEEE Transactions on Communications, vol. 57, no. 4, pp. 915 - 921, April 2009. 